\title{
Evolved ensemble of detectors for gross error detection.
}

\author{
NGUYEN, T.T., MCCALL, J., WILSON, A., OCHEI, L., CORBETT, H. and \\ STOCKTON, P.
}

2020

(C) ACM 2020. This is the author's version of the work. It is posted here for your personal use. Not for redistribution. The definitive Version of Record was published in GECCO '20: proceedings of the Genetic and evolutionary computation conference (GECCO 2020), https://doi.org/10.1145/3377929.3389906 


\title{
Evolved Ensemble of Detectors for Gross Error Detection
}

\author{
Tien Thanh Nguyen, John McCall, Allan Wilson, Laud Ochei, Helen Corbett, Phil Stockton \\ School of Computing Science and Digital Media, Robert Gordon University, United Kingdom \\ Accord Company, Aberdeen, United Kingdom \\ Aberdeen, United Kingdom \\ \{t.nguyen11,j.mccall,laud.c.ochei\}@rgu.ac.uk; \{allan.wilson,phil.stockton,helen.corbett\}@Accord-ESL.com
}

\begin{abstract}
In this study, we evolve an ensemble of detectors to check the presence of gross systematic errors on measurement data. We use the Fisher method to combine the output of different detectors and then test the hypothesis about the presence of gross errors based on the combined value. We further develop a detector selection approach in which a subset of detectors is selected for each sample. The selection is conducted by comparing the output of each detector to its associated selection threshold. The thresholds are obtained by minimizing the 0-1 loss function on training data using the Particle Swarm Optimization method. Experiments conducted on a simulated system confirm the advantages of ensemble and evolved ensemble approach.
\end{abstract}

\section{CCS CONCEPTS}

- Computing methodologies $\rightarrow$ Ensemble methods;

\section{KEYWORDS}

Gross Error Detection, Ensemble Method, Particle Swarm Optimization

\section{INTRODUCTION}

Data reconciliation has been an active area of research since the mid-1960s and has been applied to process flow measurements in, for example, the chemical, power, and oil and gas industries $[1,3]$. It is widely recognized that the techniques of reconciliation work under the assumption that only random errors (which are normally distributed measurement errors, with zero mean and known covariance) are present in the data. If non-random errors (called gross errors) caused by, for example, process disturbances, process leaks malfunctioning or miscalibrated instrumentation, or even departure from steady-state, are also present, the reconciled result can be very inaccurate and even infeasible [4]. Therefore it is important to identify gross errors before obtaining the final reconciled

Permission to make digital or hard copies of part or all of this work for personal or classroom use is granted without fee provided that copies are not made or distributed for profit or commercial advantage and that copies bear this notice and the full citation on the first page. Copyrights for third-party components of this work must be honored. For all other uses, contact the owner/author(s).

GECCO '20 Companion, July 8-12, 2020, Cancun, Mexico

(C) 2020 Copyright held by the owner/author(s).

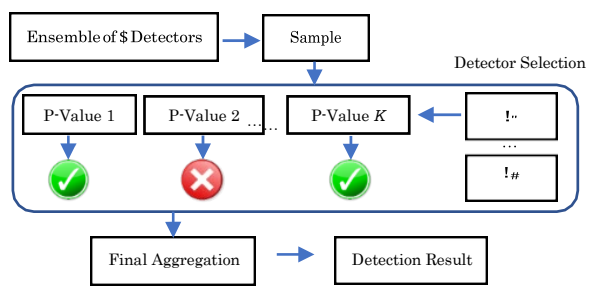

Figure 1: The proposed detector selection approach.

estimates. This study proposes an ensemble of detectors to check for the presence of gross errors. The result of each detector is combined by Fisher combination method for the collaborated detection. We further improve the performance of the proposed ensemble by searching for a suitable subset of detectors for each sample. The search process is conducted using Particle Swarm Optimization (PSO), an effective evolutionary continuous optimization method $[2,6]$.

\section{PROPOSED METHOD}

\subsection{Ensemble of Detection}

Let $\mathbf{x}_{n}$ denote a sample which is a vector of measurements obtained from the process flow and $H_{i}$ denote a detector that aims to test the null hypothesis "no gross error on $\mathbf{x}_{n}$ ". Each $H_{i}$ outputs the probability $P_{l} \times x_{n}$ (c)lled p-value) of obtaining the observed re- sults assuming that the null hypothesis is correct. In this study, we construct the ensemble of detectors $\left.H_{i} i \neq 1,\right\}$ \}., $K$ and ob- tain the collaborated detection result by combining the outputs of constituent detectors of the ensemble. The ensemble method is a popular research topic in machine learning in which the decision is made by combining the individual outputs so as to obtain a better result than each constituent member [5]. Here we use the Fisher method to combine the p-values outputted from $K$ detectors. The combination of $P_{i}\left(\mathbf{x}_{n}\right)$ on $\mathbf{x}_{n}$ is given by:

$$
S\left(\mathbf{x}_{n}\right)=-2 \times \sum_{i=1}^{K} \ln \left\{P_{i}\left(\mathbf{x}_{n}\right)\right\}
$$

When all the null hypotheses are true, and the constituent detectors are independent, $S\left(\mathbf{x}_{n}\right)$ has a chi-squared distribution: $S\left(\mathbf{x}_{n}\right) \sim$

$X_{2 \times K}^{2}$. Based on this observation, we calculate the $\mathrm{p}$-value of the combined test, denote by $P\left(\mathbf{x}_{n}\right)$. If $P\left(\mathbf{x}_{n}\right)$ is smaller than a given threshold $\alpha$ (e.g. 0.05), we reject the null hypothesis that no gross error happens on $\mathbf{x}_{n}$. The test on the combined $\mathrm{p}$-value is given by:

$$
\left\{\begin{array}{lll}
P & \left(\mathrm{x}_{n}\right)<\alpha & \text { reject the null hypothesis } \\
P & \left(\mathrm{x}_{n}\right) \geq \alpha & \text { otherwise }
\end{array}\right.
$$




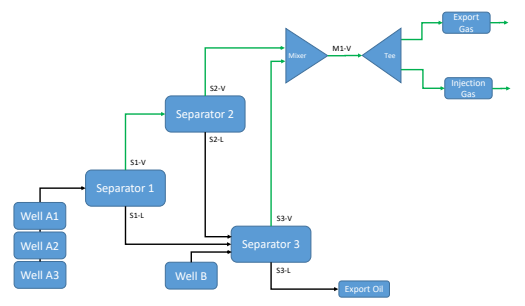

Figure 2: The hydrocarbon process plant in the experiment.

\subsection{Detector Selection Approach}

It is widely recognized that the presence of a particular detector may reduce the detection performance of the ensemble because of its poor performance or by reducing ensemble diversity. Thus, selecting a suitable subset of detectors can obtain better performance than using the whole ensemble [5]. In this study, we introduce a selection approach in which a detector is selected based on the quality of its output on each test sample. We propose using a selection threshold $\theta_{i}$ for each detector in which a detector is selected if its $\mathrm{p}$-value is smaller than its associated threshold. By this way we can select a detector based on the confidence in its prediction.

$$
\begin{cases}P_{i}\left(\mathbf{x}_{n}\right)<\theta_{i} & \text { Detector } i \text { is selected on } \mathbf{x}_{n} \\ P_{i}\left(\mathbf{x}_{n}\right) \geq \theta_{i} & \text { otherwise }\end{cases}
$$

The Fisher combination method using (3) is given by:

$$
S\left(\mathbf{x}_{n}, \boldsymbol{\theta}\right)=-2 \times \sum_{i=1}^{K} \ln \left\{P_{i}\right\} \amalg\left[P_{i}\left(\mathbf{x}_{n}\right)<\theta_{i}\right]
$$

in which $\amalg[\cdot]=1$ if the condition is true, otherwise equal 0 , $\boldsymbol{\theta}=\theta_{i}$ is the set of selection thresholds. Since $S\left(\mathbf{x}_{n}, \boldsymbol{\theta}\right) \sim \mathcal{X}_{d\left(\mathbf{x}_{n}, \boldsymbol{\theta}\right)}^{2}$ with $d\left(\mathbf{x}_{n}, \boldsymbol{\theta}\right)=2 \times \sum_{i=1}^{K} \amalg\left[P_{i}\left(\mathbf{x}_{n}\right)<\theta_{i}\right]$ degree of freedom, we can compute p-value $P\left(\mathbf{x}_{n}, \boldsymbol{\theta}\right)$ of the test. We apply (2) with $P\left(\mathbf{x}_{n}, \boldsymbol{\theta}\right)$ to determine whether the null hypothesis is rejected. One question arises from this model is to search for the selection threshold $\boldsymbol{\theta}$. In this study, we minimize the $0-1$ loss function on the training data (with $N$ observations) to find the optimal value for $\theta$. The optimization problem is given by:

$$
\begin{gathered}
\min _{\theta}\left\{1-\frac{1}{N} \sum_{n=1}^{N} \amalg\left[\amalg\left[P\left(\mathbf{x}_{n}, \boldsymbol{\theta}\right)<\alpha\right]=y_{n}\right]\right\} \\
\text { s.t. } \theta_{i} \in[0,1] ; i=1, \ldots, K
\end{gathered}
$$

in which $y_{n} \in\{0,1\}$ is the ground truth of $\mathbf{x}_{n}$. We use PSO [2] to solve the problem in (5) because of its advantages in solving the optimization problem [6]. The position of each particle encodes $\boldsymbol{\theta}$ while the fitness is the value of the loss function calculated by using $\boldsymbol{\theta}$ on the training data. Through a number of iterations, we obtain the optimal value for $\boldsymbol{\theta}$.

\section{EXPERIMENTAL STUDIES}

\subsection{Dataset and Settings}

We consider a hydrocarbon process plant consisting of four production wells and nine streams in the process conditioning the fluids for transportation (see Fig.2). A process simulation package called
Table 1: The experimental results

\begin{tabular}{lccccc}
\hline \multirow{2}{*}{ Detectors } & \multicolumn{2}{c}{ Test Case 1 } & & \multicolumn{2}{c}{ Test Case 2 } \\
\cline { 2 - 3 } \cline { 5 - 6 } & Accuracy & 1 1 Score & & Accuracy & F1 Score \\
\hline Global Test & 0.2081 & 0.1887 & & 0.2683 & 0.2666 \\
PCA Test & 0.1832 & 0.1685 & & 0.2494 & 0.2440 \\
Measurement Test & 0.4737 & 0.3545 & & 0.5017 & 0.4446 \\
Constraint Test & 0.3277 & 0.2713 & & 0.3733 & 0.3547 \\
GLR Test & 0.4441 & 0.3390 & & 0.4772 & 0.4290 \\
Ensemble & 0.5253 & 0.3786 & & 0.5422 & 0.4650 \\
Evolved Ensemble & $\mathbf{0 . 5 8 7 3}$ & $\mathbf{0 . 4 0 7 3}$ & & $\mathbf{0 . 5 8 5 0}$ & $\mathbf{0 . 4 8 7 5}$ \\
\hline
\end{tabular}

CHARM ${ }^{1}$ was applied to calculate the flow rates for the streams. This gave a vector for measurements of each of the streams, indicating that no gross errors are present. The gross errors were generated by randomly changing the magnitude of any one of the four wells and two internal process streams by $+5 \%$ and $+25 \%$.

We created a training dataset including 800 observations with no gross error and 800 observations with a gross error. We also created two test cases containing 7400 and 1800 observations respectively. We used five detectors namely: Global Test, PCA Test, Measurement Test, Constraint Test, and GLR Test [4] to construct the ensemble. The performances of all detectors were reported in terms of the detection accuracy and the F1 score. For the PSO algorithm, the maximum number of iterations was set to 200 , the population size was set to 500 , and social attraction and cognitive attraction were set to 1.494 .

\subsection{Result and Discussions}

Table 1 shows the detection accuracy and F1 score of five detectors and the ensemble of detectors. Some observations can be made:

- The PCA test is the poorest detectors: it obtains the lowest results of detection accuracy ( 0.1832 and 0.2494$)$ and F1 score (0.1685 and 0.2480) among five methods in both test cases. In contrast, Measurement Test performs best among all five constituent detectors.

- The use of an ensemble approach can improve the performance on both measures. The ensemble approach is about $4.5 \%$ and $2 \%$ better than the top detector i.e. Measurement Test for detection accuracy and F1 score, respectively in both test cases

- The proposed evolutionary selection of detectors can improve the performance of the ensemble. For example, the evolved ensemble achieves about $6 \%$ and $3 \%$ better than the ensemble for detection accuracy and F1 score, respectively in test case 1 .

\section{REFERENCES}

[1] Xiaolong Jiang, Pei Liu, and Zheng Li. 2014. Data reconciliation and gross error detection for operational data in power plants. Energy 75 (2014), 14-23.

[2] James Kennedy and Russell Eberhart. 1995. Particle swarm optimization. In Proceedings of ICNN'95-International Conference on Neural Networks, Vol. 4. IEEE, 1942-1948.

[3] José Loyola-Fuentes and Robin Smith. 2019. Data reconciliation and gross error detection in crude oil pre-heat trains undergoing shell-side and tube-side fouling deposition. Energy 183 (2019), 368-384.

[4] Shankar Narasimhan and Cornelius Jordache. 1999. Data reconciliation and gross error detection: An intelligent use of process data. Elsevier.

[5] Tien Thanh Nguyen, Anh Vu Luong, Manh Truong Dang, Alan Wee-Chung Liew, and John McCall. 2020. Ensemble Selection based on Classifier Prediction Confidence. Pattern Recognition 100 (2020), 107104.

[6] RE 1 Perez and K Behdinan. 2007. Particle swarm approach for structural design optimization. Computers \& Structures 85, 19-20 (2007), 1579-1588.

${ }^{1}$ https://charm.accord-esl.com/Charm/ 